\title{
Norm inequalities for the conjugate operator in two-weighted Lebesgue spaces
}

Kyung Soo $\operatorname{Rim}^{*}$ and Jaesung Lee

* Correspondence: ksrim@sogang. ac.kr

Department of Mathematics, Sogang University, Seoul 121-742, Korea

\section{Abstract}

In this article, first, we prove that weighted-norm inequalities for the M-harmonic conjugate operator on the unit sphere whenever the pair $(u, v)$ of weights satisfies the $A_{p}$-condition, and $u d \sigma, v d \sigma$ are doubling measures, where $d \sigma$ is the rotationinvariant positive Borel measure on the unit sphere with total measure 1. Then, we drive cross-weighted norm inequalities between the Hardy-Littlewood maximal function and the sharp maximal function whenever $(u, v)$ satisfies the $A_{p}$-condition, and $v d \sigma$ does a certain regular condition.

2000 MSC: primary 32A70; secondary 47G10.

Keywords: two-weighted norm inequality, non-isotropic metric, maximal function, sharp maximal function, M-harmonic conjugate operator, Hilbert transform

\section{Introduction}

Let $B$ be the unit ball of $\mathbb{C}^{n}$ with norm $|z|=\langle z, z\rangle^{1 / 2}$ where $\langle$,$\rangle is the Hermitian inner$ product, $S$ be the unit sphere and $\sigma$ be the rotation-invariant probability measure on $S$.

For $z \in B, \xi \in S$, we define the $\mathcal{M}$-harmonic conjugate kernel $K(z, \xi)$ by

$$
i K(z, \xi)=2 C(z, \xi)-P(z, \xi)-1,
$$

where $C(z, \xi)=(1-\langle z, \xi\rangle)^{-n}$ is the Cauchy kernel and $P(z, \xi)=\left(1-|z|^{2}\right)^{n} /|1-\langle z, \xi\rangle|$ ${ }^{2 n}$ is the invariant Poisson kernel [1].

For the kernels, $C$ and $P$, refer to [2]. And for all $f-A(B)$, the ball algebra, such that $f$ (0) is real, the reproducing property of $2 C(z, \xi)-1$ [2, Theorem 3.2.5] gives

$$
\int_{S} K(z, \xi) \operatorname{Re} f(\xi) d \sigma(\xi)=-i(f(z)-\operatorname{Re} f(z))=\operatorname{Im} f(z) .
$$

For $n=1$, the definition of $K f$ is the same as the classical harmonic conjugate function and so we can regard $K f$ as the Hilbert transform on the unit circle. The $L^{p}$ boundedness property of harmonic conjugate functions on the unit circle for $1<p<\infty$ was introduced by Riesz in 1924 [3, Theorem 2.3 of Chapter 3]. Later, in 1973, Hunt et al. [4] proved that, for $1<p<\infty$, conjugate functions are bounded on weighted measured Lebesgue space if and only if the weight satisfies $A_{p}$-condition. Most recently, Lee and Rim [5] provided an analogue of that of [4] by proving that, for $1<p<\infty$, Mharmonic conjugate operator $K$ is bounded on $L^{p}(\omega)$ if and only if the nonnegative weight $\omega$ satisfies the $A_{p}(S)$-condition on $S$; i.e., the nonnegative weight $\omega$ satisfies

(C) 2011 Rim and Lee; licensee Springer. This is an Open Access article distributed under the terms of the Creative Commons Attribution License (http://creativecommons.org/licenses/by/2.0), which permits unrestricted use, distribution, and reproduction in any medium, provided the original work is properly cited. 


$$
\sup _{Q} \frac{1}{\sigma(Q)} \int_{Q} \omega d \sigma\left(\frac{1}{\sigma(Q)} \int_{Q} \omega^{-1 /(p-1)} d \sigma\right)^{p-1}:=A_{p}^{\omega}<\infty
$$

where $Q=Q(\xi, \delta)=\left\{\eta \in S: d(\xi, \eta)=|1-\langle\xi, \eta\rangle|^{1 / 2}<\delta\right\}$ is a non-isotropic ball of $S$.

To define the $A_{p}(S)$-condition for two weights, we let $(u, v)$ be a pair of two nonnegative integrable functions on $S$. For $p>1$, we say that $(u, v)$ satisfies two-weighted $A_{p}(S)$-condition if

$$
\sup _{Q} \frac{1}{\sigma(Q)} \int_{Q} u d \sigma\left(\frac{1}{\sigma(Q)} \int_{Q} v^{-1 /(p-1)} d \sigma\right)^{p-1}:=A_{p}<\infty
$$

where $Q$ is a non-isotropic ball of $S$. For $p=1$, the $A_{1}(S)$-condition can be viewed as a limit case of the $A_{p}(S)$-condition as $p \searrow 1$, which means that $(u, v)$ satisfies the $A_{1}$ (S)-condition if

$$
\sup _{Q} \frac{1}{\sigma(Q)} \int_{Q} u d \sigma\left(\operatorname{essup}_{Q} v^{-1}\right):=A_{1}<\infty,
$$

where $Q$ is a non-isotropic ball of $S$.

In succession of classical weighted-norm inequalities, starting from Muckenhoupt's result in 1975 [6], there have been extensive studies on two-weighted norm inequalities (for textbooks [7-10] and for related topics [11-17]). In [6], Muckenhoupt derives a necessary and sufficient condition on two-weighted norm inequalities for the Poisson integral operator. And then, Sawyer [18,19] obtained characterizations of two-weighted norm inequalities for the Hardy-Littlewood maximal function and for the fractional and Poisson integral operators, respectively. As a result on two-weighted $A_{p}(S)$-condition itself, Neugebauer [20] proved the existence of an inserting pair of weights. CruzUribe and Pérez [21] give a sufficient condition for Calderón-Zygmund operators to satisfy the weighted weak $(p, p)$ inequality. More recently, Martell et al. [22] provide two-weighted norm inequalities for Calderón-Zygmund operators that are sharp for the Hilbert transform and for the Riesz transforms.

Ding and Lin [23] consider the fractional integral operator and the maximal operator that contain a function homogeneous of degree zero as a part of kernels and the authors prove weighted $\left(L^{p}, L^{q}\right)$-boundedness for those operators for two weights.

In [24], Muckenhoupt and Wheeden provided simple examples of a pair that satisfies two-weighted $A_{p}(\mathbb{R})$-condition but not two-weighted norm inequalities for the HardyLittlewood maximal operator and the Hilbert transform. In this article, we prove the converse of the main theorem of [5] by adding a doubling condition for a weight function. And then by adding a suitable regularity condition on a weight function, we derive and prove a cross-weighted norm inequalities between the Hardy-Littlewood maximal function and the sharp maximal function.

Throughout this article, $Q$ denotes a non-isotropic ball of $S$ induced by the non-isotropic metric $d$ on $S$ which is defined by $d(\xi, \eta)=|1-\langle\xi, \eta\rangle|^{1 / 2}$. For notational simplicity, we denote $\otimes_{Q} f d \sigma:=f(Q)$ the integral of $f$ over $Q$, and $\frac{1}{\sigma(Q)} \int_{Q} f d \sigma:=f_{Q}$ the average of $f$ over $Q$. Also, for a nonnegative integrable function $u$ and a measurable subset $E$ of $S$, we write $u(E)$ for the integral of $u$ over $E$. We write $Q(\delta)$ in place of $Q(\xi$, $\delta$ ) whenever the center $\xi$ has no meaning in a context. For a positive constant $s, s Q(\delta)$ 
means $Q(s \delta)$. We say that a weight $v$ satisfies a doubling condition if there is a constant $C$ independent of $Q$ such that $v(2 Q) \leq C v(Q)$ for all $Q$.

Theorem 1.1. Let $1<p<\infty$. If $(u, v)$ satisfies two-weighted $A_{p^{\prime}}(S)$-condition for some $p^{\prime}<p$ and $u d \sigma, v d \sigma$ are doubling measures, then there exists a constant $C$ which depends on $u, v$ and $p$, such that for all function $f$,

$$
\int_{S}|K f|^{p} u d \sigma \leq C \int_{S}|f|^{p} v d \sigma \quad \text { for all } f \in L^{p}(v) .
$$

To prove the next theorem, we need a regularity condition for $v$ such that for $1 \leq p$ $<\infty$, we assume that for a measurable set $E \subset Q$ and for $\sigma(E) \leq \theta \sigma(Q)$ with $0 \leq \theta \leq 1$, we get

$$
v(E) \leq\left(1-(1-\theta)^{p}\right) v(Q) .
$$

Let $f \in L^{1}(S)$ and let $1<p<\infty$. The (Hardy-Littlewood) maximal and the sharp maximal functions $M f$, $f^{* p}$, resp. on $S$ are defined by

$$
\begin{aligned}
& M f(\xi)=\sup _{Q} \frac{1}{\sigma(Q)} \int_{Q}|f| d \sigma, \\
& f^{\# p}(\xi)=\sup _{Q}\left(\frac{1}{\sigma(Q)} \int_{Q}\left|f-f_{Q}\right|^{p} d \sigma\right)^{1 / p},
\end{aligned}
$$

where each supremum is taken over all balls $Q$ containing $\xi$. From the definition, the sharp maximal function $f \mapsto f^{\# p}$ is an analogue of the maximal function $M f$, which satisfies $f^{\# 1}(\xi) \leq 2 M f(\xi)$.

Theorem 1.2. Let $1<p<\infty$. If $(u, v)$ satisfies two-weighted $A_{p}(S)$-condition and $v d \sigma$ does (1.3), then there exists a constant $C$ which depends on $u, v$ and $p$, such that for all function $f$,

$$
\int_{S}(M f)^{p} u d \sigma \leq C\left(\int_{S}\left(f^{\# 1}\right)^{p} v d \sigma+\int_{S}|f|^{p} v d \sigma\right) .
$$

Remark. On the unit circle, we derive a sufficient condition for weighted-norm inequalities for the Hilbert transform for two weights.

The proofs of Theorem 1.1 will be given in Section 3. We start Section 2 by deriving some preliminary properties of $(u, v)$ which satisfies the $A_{p}(S)$-condition. In Section 4, we prove Theorem 1.2.

\section{Two-weight on the unit sphere}

Lemma 2.1. If $(u, v)$ satisfies two-weighted $A_{p}(S)$-condition, then for every function $f \geq 0$ and for every ball $Q$,

$$
\left(f_{Q}\right)^{p} u(Q) \leq A_{p} \int_{Q} f^{p} v d \sigma .
$$

Proof. If $p=1$ and $(u, v)$ satisfies two-weighted $A_{1}(S)$-condition, we get, for every ball $Q$ and every $f \geq 0$, 


$$
\begin{aligned}
f_{Q} u(Q) & =f(Q) u_{Q} \\
& \leq A_{1} f(Q) \frac{1}{\operatorname{ess} \sup v^{-1}} \\
& \leq A_{1} \int_{Q} f v d \sigma,
\end{aligned}
$$

since $1 / \operatorname{ess} \sup v^{-1}=\underset{Q}{\operatorname{ess} \inf v} \leq(\xi)$ for all $\xi \in Q$.

If $1<p<\infty$ and $(u, v)$ satisfies two-weighted $A_{p}(S)$-condition, we have, for every ball $Q$ and every $f \geq 0$, using Holder's inequality with $p$ and its conjugate exponent $p /(p$ $1)$,

$$
\begin{aligned}
f_{Q} & =\frac{1}{\sigma(Q)} \int_{Q} f v^{1 / p} v^{-1 / p} d \sigma \\
& \leq\left(\frac{1}{\sigma(Q)} \int_{Q} f^{p} v d \sigma\right)^{1 / p}\left(\frac{1}{\sigma(Q)} \int_{Q} v^{-1 /(p-1)} d \sigma\right)^{(p-1) / p}
\end{aligned}
$$

Hence,

$$
\begin{aligned}
\left(f_{Q}\right)^{p} u(Q) & =\frac{u(Q)}{\sigma(Q)}\left(\frac{1}{\sigma(Q)} \int_{Q} v^{-1 /(p-1)} d \sigma\right)^{p-1} \int_{Q} f^{p} v d \sigma \\
& \leq A_{p} \int_{Q} f^{p} v d \sigma .
\end{aligned}
$$

Therefore, the proof is complete.

Corollary 2.2. If $(u, v)$ satisfies two-weighted $A_{p}(S)$-condition, then

$$
\left(\frac{\sigma(E)}{\sigma(Q)}\right)^{p} u(Q) \leq A_{p} v(E)
$$

where $E$ is a measurable subset of $Q$.

Proof. Applying Lemma 2.1 with $f$ replaced by $\chi_{E}$ proves the conclusion.

\section{Proof of Theorem 1.1}

In this section, we will prove the first main theorem. First, we derive the inequality between two sharp maximal functions of $K f$ and $f$.

Lemma 3.1. Let $f \in L^{1}(S)$. Then, for $q>p>1$, there is a constant $C_{p, q}$ such that $(K f)$ $\# p(\xi) \leq C_{p, q} f^{\# q}(\xi)$ for almost every $\xi$.

Proof. It suffices to show that for $r \geq 1$ and $q>1$, there is a constant $C_{r q}$ such that ( $K$ $f)^{\# r}(\xi) \leq C_{r q} f^{\# r q}(\xi)$ for almost every $\xi$,

i.e., for $Q=Q\left(\xi_{Q}, \delta\right)$ a ball of $S$, we prove that there are constants $\lambda=\lambda(Q, f)$ and $C_{r q}$ such that

$$
\left(\frac{1}{\sigma(Q)} \int_{Q}|K f(\eta)-\lambda|^{r} d \sigma\right)^{1 / r} \leq C_{r, q} f^{\# q^{\prime}}\left(\xi_{Q}\right) .
$$

Fix $Q=Q\left(\xi_{Q}, \delta\right)$ and write

$$
\begin{aligned}
f(\eta) & =\left(f(\eta)-f_{Q}\right) \chi_{2 Q}(\eta)+\left(f(\eta)-f_{Q}\right) \chi_{S \backslash 2 Q}(\eta)+f_{Q} \\
& :=f_{1}(\eta)+f_{2}(\eta)+f_{Q} .
\end{aligned}
$$


Then, $K f=K f_{1}+K f_{2}$, since $K f_{Q}=0$.

For each $z \in B$, put

$$
g(z)=\int_{S}(2 C(z, \xi)-1) f_{2}(\xi) d \sigma(\xi) .
$$

Then, $g$ is continuous on $B \cup Q$ By setting $\lambda=-i g\left(\xi_{Q}\right)$ in (3.1), we shall drive the conclusion. By Minkowski's inequality, we split the integral in (3.1) into two parts,

$$
\begin{aligned}
& \left(\frac{1}{\sigma(Q)} \int_{Q}\left|K f(\eta)+i g\left(\xi_{Q}\right)\right|^{r} d \sigma(\eta)\right)^{1 / r} \\
& \quad \leq\left(\frac{1}{\sigma(Q)} \int_{Q}\left|K f_{1}\right|^{r} d \sigma\right)^{1 / r}+\left(\frac{1}{\sigma(Q)} \int_{Q}\left|K f_{2}+i g\left(\xi_{Q}\right)\right|^{r} d \sigma\right)^{1 / r}:=I_{1}+I_{2} .
\end{aligned}
$$

We estimate $I_{1}$. By Holder's inequality, it is estimated as

$$
\begin{aligned}
I_{1} & \leq\left(\frac{1}{\sigma(Q)} \int_{Q}\left|K f_{1}\right|^{r q} d \sigma\right)^{1 / r q} \\
& \leq\left(\frac{1}{\sigma(Q)} \int_{S}\left|K f_{1}\right|^{r q} d \sigma\right)^{1 / r q} \leq \frac{C_{r q}}{\sigma(Q)^{1 / r q}}\left\|f_{1}\right\|_{L^{r q}}
\end{aligned}
$$

since $K$ is bounded on $L^{r q}(S)(r q>1)$. By replacing $f_{1}$ by $f$ - $f_{\mathrm{Q}}$, we get

$$
\begin{aligned}
\left\|f_{1}\right\|_{L^{r q}} & =\left(\int_{2 Q}\left|f-f_{Q}\right|^{r q} d \sigma\right)^{1 / r q} \\
& \leq\left(\int_{2 Q}\left|f-f_{2 Q}\right|^{r q} d \sigma\right)^{1 / r q}+\sigma(2 Q)^{1 / r q}\left|f_{2 Q}-f_{Q}\right| .
\end{aligned}
$$

Thus, by applying Hölder's inequality in the last term of the above,

$$
\begin{aligned}
\sigma(2 Q)^{1 / r q}\left|f_{2 Q}-f_{Q}\right| & \leq \frac{\sigma(2 Q)^{1 / r q}}{\sigma(Q)} \int_{Q}\left|f-f_{2 Q}\right| d \sigma \\
& \leq \frac{\sigma(2 Q)^{1 / r q} \sigma(Q)^{1-1 / r q}}{\sigma(Q)}\left(\int_{2 Q}\left|f-f_{2 Q}\right|^{r q} d \sigma\right)^{1 / r q} \\
& =R_{2}^{1 / r q}\left(\int_{2 Q}\left|f-f_{2 Q}\right|^{r q} d \sigma\right)^{1 / r q}(\operatorname{by}(4.2)) .
\end{aligned}
$$

Hence,

$$
I_{1} \leq C_{r q}\left(1+R_{2}^{1 / r q}\right) f^{\# r q}\left(\xi_{Q}\right) .
$$

Now, we estimate $I_{2}$. Since $f_{2} \equiv 0$ on $2 Q$, the invariant Poisson integral of $f_{2}$ vanishes on $Q$, i.e., $\lim _{t, 1} \int_{S} P(t \eta, \xi) f_{2}(\eta) d \sigma(\eta)=0$ whenever $\xi \in Q$. Thus, for almost all $\xi \in Q$,

$$
i K f_{2}(\xi)=\int_{S \backslash 2 Q}(2 C(\xi, \eta)-1) f_{2}(\eta) d \sigma(\eta)=g(\xi)
$$


and then, by Minkowski's inequality for integrals,

$$
\begin{aligned}
I_{2} & =\left(\frac{1}{\sigma(Q)} \int_{Q}\left|i K f_{2}-g\left(\xi_{Q}\right)\right|^{r} d \sigma\right)^{1 / r} \\
& \leq \int_{S \backslash 2 Q} 2\left|f_{2}(\eta)\right|\left(\frac{1}{\sigma(Q)} \int_{Q}\left|C(\xi, \eta)-C\left(\xi_{Q}, \eta\right)\right|^{r} d \sigma(\xi)\right)^{1 / r} d \sigma(\eta) .
\end{aligned}
$$

By Lemma 6.1.1 of [2], we get an upper bound such that

$$
I_{2} \leq C \delta \int_{S \backslash 2 Q} \frac{\left|f_{2}(\eta)\right|}{\left|1-\left\langle\eta, \xi_{Q}\right\rangle\right|^{n+1 / 2}} d \sigma(\eta),
$$

where $C$ is an absolute constant. Write $S \backslash 2 Q=\bigcup_{k=1}^{\infty} 2^{k+1} Q \backslash 2^{k} Q$. Then, the integral of (3.4) is equal to

$$
\begin{aligned}
& \sum_{k=1}^{\infty} \int_{2^{k+1} Q \backslash 2^{k} Q} \frac{\left|f(\eta)-f_{Q}\right|}{\left|1-\left\langle\eta, \xi_{Q}\right\rangle\right|^{n+1 / 2}} d \sigma(\eta) \\
& \leq \sum_{k=1}^{\infty} \frac{1}{2^{(2 n+1) k \delta^{2 n+1}}} \int_{2^{k+1} Q \backslash 2^{k} Q}\left|f-f_{Q}\right| d \sigma \\
& \leq \sum_{k=1}^{\infty} \frac{1}{2^{(2 n+1) k \delta^{2 n+1}}}\left(\int_{2^{k+1} Q}\left|f-f_{2^{k+1} Q}\right| d \sigma+\sum_{j=0}^{k} \int_{2^{k+1} Q}\left|f_{2^{j+1} Q}-f_{2^{j} Q}\right| d \sigma\right) .
\end{aligned}
$$

By Hölder's inequality, by (4.3),

$$
\begin{aligned}
\int_{2^{k+1} Q}\left|f-f_{2^{k+1} Q}\right| d \sigma & \leq R_{2^{k+1} \delta}\left(\frac{1}{\sigma\left(2^{k+1} Q\right)} \int_{2^{k+1} Q}\left|f-f_{2^{k+1} Q}\right|^{r q} d \sigma\right)^{1 / r q} \\
& \leq R_{2^{k+1} \delta} f^{\# r^{r q}}\left(\xi_{Q}\right),
\end{aligned}
$$

Similarly, for each $j$,

$$
\begin{aligned}
& \int_{2^{k+1} Q}\left|f_{2^{j+1} Q}-f_{2^{j} Q}\right| d \sigma \leq \frac{\sigma\left(2^{k+1} Q\right)}{\sigma\left(2^{j} Q\right)} \int_{2^{j} Q}\left|f-f_{2^{j+1} Q}\right| d \sigma \\
& \leq R_{2^{k-j+1}} \int_{2^{j+1} Q}\left|f-f_{2^{j+1} Q}\right| d \sigma \quad \text { (by (4.2)) } \\
& \leq R_{2^{k-j+1}} R_{2^{j+1} \delta} f^{\# t q}\left(\xi_{Q}\right) \quad \text { (from (3.5) with } k=j \text { ) } \\
& =R_{1} R_{2^{k+2} \delta} f^{\# r q}\left(\xi_{Q}\right) \text {. }
\end{aligned}
$$

Thus,

$$
\sum_{j=0}^{k} \int_{2^{k+1} Q}\left|f_{2^{j+1} Q}-f_{2^{j} Q}\right| d \sigma \leq(k+1) R_{1} R_{2^{k+2} \delta} f^{\#^{r q}}\left(\xi_{Q}\right) .
$$

Since $R_{s}$ increases as $s \nearrow \infty$ and $R_{1}>1$, by adding (3.5) to (3.6), we have the upper bound as

$$
(k+2) R_{1} R_{2^{k+2} \delta} f^{\# q q}\left(\xi_{Q}\right) .
$$


Eventually, the identity of $R_{2^{k+2} \delta}=R_{1} 2^{2 n(k+2)} \delta^{2 n}$ yields that

$$
I_{2} \leq 2^{4 n} C R_{1}^{2} \sum_{k=1}^{\infty} \frac{k+2}{2^{k}} f^{\# r q}\left(\xi_{Q}\right),
$$

and therefore, combining (3.3) and (3.7), we complete the proof.

The main theorem depends on Marcinkiewicz interpolation theorem between two abstract Lebesgue spaces, which is as follows.

Proposition 3.2. Suppose $(X, \mu)$ and $(Y, v)$ are measure spaces; $p_{0}, p_{1}, q_{0}, q_{1}$ are elements of $[1, \infty]$ such that $p_{0} \leq q_{0}, p_{1} \leq q_{1}$ and $q_{0} \neq q_{1}$ and

$$
\frac{1}{p}=\frac{1+t}{p_{0}}+\frac{t}{p_{1}}, \quad \frac{1}{q}=\frac{1-t}{q_{0}}+\frac{t}{q_{1}} \quad(0<t<1) .
$$

If $T$ is a sublinear map from $L^{p_{0}}(\mu)+L^{p_{1}}(\mu)$ to the space of measurable functions on $Y$ which is of weak-types $\left(p_{0}, q_{0}\right)$ and $\left(p_{1}, q_{1}\right)$, then $T$ is of type $(p, q)$.

Now, we prove the main theorem.

Proof of Theorem 1.1. Under the assumption of the main theorem, we will prove that (1.2) holds. We fix $p>1$ and let $f \in L^{p}(v)$.

By Theorem 1.2, there is a constant $C_{p}$ such that

$$
\begin{aligned}
\int_{S}|K f|^{p} u d \sigma & \leq \int_{S}\left|M_{u}(K f)\right|^{p} u d \sigma \\
& \leq C_{p} \int_{S}\left|(K f)^{\#^{1}}\right|^{p} u d \sigma \\
& \left.\leq C_{p} \int_{S}\left|f^{\#^{q}}\right|^{p} u d \sigma \quad \text { (by Lemma 3.1 with } q>1, p / q>1\right) \\
& \leq 2^{p} C_{p} \int_{S}\left(M|f|^{q}\right)^{p / q} u d \sigma \quad \text { (by the triangle inequality). }
\end{aligned}
$$

where $M_{u}$ is the maximal operator with respect to $u d \sigma$, the second inequality follows from the doubling condition of $u d \sigma$.

Without loss of generality, we assume $f \geq 0$. By Holder's inequality and by (1.1), we have

$$
\begin{aligned}
\frac{1}{\sigma(Q)} \int_{Q} d \sigma & \leq\left(\frac{1}{\sigma(Q)} \int_{Q} f^{p / q} v d \sigma\right)^{q / p}\left(\frac{1}{\sigma(Q)} \int_{Q} v^{-1 /(p / q-1)} d \sigma\right)^{1-q / p} \\
& \leq A_{p / q}^{q / p}\left(\frac{1}{\sigma(Q)} \int_{Q} f^{p / q} v d \sigma\right)^{q / p}\left(\frac{\sigma(Q)}{v(Q)}\right)^{q / p} \text { for all } Q
\end{aligned}
$$

Thus, if $f_{Q}>\lambda$, then

$$
u(Q) \leq A_{p / q} \lambda^{p / q} \int_{Q} f^{p / q} v d \sigma \quad \text { for all } Q
$$

Let $E$ be an arbitrary compact subset of $\{\xi \in S: M f(\xi)>\lambda\}$. Since $v d \sigma$ is a doubling measure, from (3.9), there exists a constant $C_{p, q}$ such that

$$
u(E) \leq C_{p, q} \lambda^{p / q} \int_{S} f^{p / q} v d \sigma .
$$


Thus, $M f$ is of weak-type $\left(L^{p / q}(v d \sigma), L^{p / q}(u d \sigma)\right)$. Moreover,

$$
\begin{aligned}
\|M f\|_{L^{\infty}(u d \sigma)} & \leq\|M f\|_{L^{\infty}} \quad(\text { since } u d \sigma \ll d \sigma) \\
& \leq\|f\|_{L^{\infty}} \\
& \left.=\|f\|_{L^{\infty}(u d \sigma)} \quad \text { (since } u d \sigma \ll d \sigma, v>0 \text { a.e. by }(1.1)\right) .
\end{aligned}
$$

Now, by Proposition 3.2, $M f$ is of type $\left(L^{r}(v d \sigma), L^{r}(u d \sigma)\right)$ for $f>p / q$.

Hence, the last integral of (3.8) is bounded by some constant times

$$
\int_{S}|f|^{q r} v d \sigma \quad(\text { for all } r>p / q) \text {. }
$$

Since $q$ is arbitrary so that $p / q>0$, we can replace $q r$ by $p$ with $p>1$. Therefore, the proof is completed.

\section{Proof of Theorem 1.2}

Theorem 1.2 can be regarded as cross-weighted norm inequalities for the Hardy-Littlewood maximal function and the sharp maximal function on the unit sphere. For a single $A_{p}$-weight in $\mathbb{R}^{n}$, refer to Theorem 2.20 of [8].

From Proposition 5.1.4 of [2], we conclude that when $n>1$,

$$
\frac{\Gamma^{2}(n / 2+1)}{2^{n-2} \Gamma(n+1)} s^{2 n} \leq \frac{\sigma(Q(s \delta))}{\sigma(Q(\delta))} \leq \frac{2^{n-2} \Gamma(n+1)}{\Gamma^{2}(n / 2+1)} s^{2 n},
$$

and when $n=1$,

$$
\frac{2}{\pi} s^{2} \leq \frac{\sigma(Q(s \delta))}{\sigma(Q(\delta))} \leq \frac{\pi}{2} s^{2}
$$

for any $s>0$. Throughout the article, several kinds of constants will appear. To avoid confusion, we define the maximum ratio between sizes of two balls by

$$
R_{s}: R_{s, n}=\max \left(\frac{2^{n-2} \Gamma(n+1)}{\Gamma^{2}(n / 2+1)}, \frac{\pi}{2}\right) s^{2 n},
$$

and thus, for every $s>0$, for every $\delta>0$,

$$
\sigma(s Q(\delta)) \leq R_{s} \sigma(Q(\delta)) .
$$

Putting $\delta=1$ in (4.2), we get

$$
\sigma(Q(s)) \leq R_{s} .
$$

To prove Theorem 1.2, we need some lemmas. The next result is a covering lemma on the unit sphere, related to the maximal function. Let $f \in L^{1}(S)$ and let $t>\|f\|_{L^{1}(S)}$. We may assume $\|f\|_{L^{1}(S)} \neq 0$. Since $\{M f>t\}$ is open, take a ball $Q \subset\{M f>t\}$ with center at each point of $\{M f>t\}$. For such a ball $Q$,

$$
\sigma(Q) \leq \frac{1}{t} \int_{Q}|f| d \sigma
$$

Thus, to each $\xi \in\{M f>t\}$ corresponds a largest radius $\delta$ such that the ball $Q=Q(\xi$, $\delta) \subset\{M f>t\}$ satisfies (4.4). Hence, we conclude the following simple covering lemma. 
Lemma 4.1 (Covering lemma on $S$ ). Let $f \in L^{1}(S)$ be non-trivial. Then, for $t>\|f\|_{L^{1}(S)}$, there is a collection of balls $\left\{Q_{t, j}\right\}$ such that

(i) $\{\xi \in S: M f(\xi)>t\} \subset \bigcup_{j} Q_{t, j}$,

(ii) $\sigma\left(Q_{t, j}\right) \leq t^{-1}|f|\left(Q_{t, j}\right)$,

where each $Q_{t, j}$ has the maximal radius of all the balls that satisfy (ii) in the sense that if $Q$ is a ball that contains some $Q_{t, j}$ as its proper subset, then $\sigma(Q)>t^{-1} \Xi_{Q}|f| d \sigma$ holds.

Now, we are ready to prove Theorem 1.2.

Proof of Theorem 1.2. Fix $1<p<\infty$. We may assume $f^{11} \in L^{p}(v)$ and $f \in L^{p}(v)$, otherwise, Theorem 1.2 holds clearly. Since $v$ satisfies the doubling condition, we have $\| M$ $\left.f\right|_{L p(v)} \leq C|| f^{\sharp 1} \|_{L p(v)}$. Combining this with $f^{\sharp 1} \in L^{p}(v)$, we have $\|M f\|_{L p(v)}<\infty$.

Suppose that $f$ is non-trivial and we may assume that $f \geq 0$. Let

$$
t>\max \left(2,2 R_{2}^{2}, R_{3}\right)\|f\|_{L^{1}(S)} .
$$

For $\varepsilon>0, E_{t}$ be a compact subset of $\{M f>t\}$ such that $u(\{M f>t\})<u\left(E_{t}\right)+e^{-t} \varepsilon$. Indeed, since $u$ is integrable, $u d \sigma$ is a regular Borel measure absolutely continuous with respect to $\sigma$.

Suppose $\left\{Q_{t, j}\right\}$ is a collection of balls having the properties (i) and (ii) of Lemma 4.1. Since $\left\{Q_{t, j}\right\}$ is a cover of a compact set $E_{t}$, there is a finite subcollection of $\left\{Q_{t, j}\right\}$, which covers $E_{t}$. By Lemma 5.2.3 of [2], there are pairwise disjoint balls, $Q_{t, j_{1}}, Q_{t, j_{2}}, \ldots, Q_{t, j e}$ of the previous subcollection such that

$$
\begin{gathered}
E_{t} \subset \bigcup_{k=1}^{\ell} 3 Q_{t, j_{k^{\prime}}} \\
\sigma\left(E_{t}\right) \leq R_{3} \sum_{k=1}^{\ell} \sigma\left(Q_{t, j_{k}}\right),
\end{gathered}
$$

where $\ell$ may depend on $t$. To avoid the abuse of subindices, we rewrite $Q_{t, j_{k}}$ as $Q_{t, j}$. Let us note that from the maximality of $Q_{t, j}$,

$$
t>\frac{1}{\sigma\left(2 Q_{t . j}\right)} \int_{2 Q_{t, j}} f d \sigma \geq \frac{\sigma\left(Q_{t . j}\right)}{\sigma\left(2 Q_{t . j}\right)} t \geq R_{2}^{-1} t .
$$

Fix $Q_{0}=2 Q_{k t, j o}$, where $\kappa^{-1}=2 R_{2}$. (Here, $\kappa<1 / 2$, since $R_{2}>1$.) Let $\lambda>0$ that will be chosen later. From the definition of the sharp maximal function, there are two possibilities: either

$$
Q_{0} \subset\left\{f^{\# 1}>\lambda t\right\} \quad \text { or } \quad Q_{0} \not \subset\left\{f^{\#^{1}}>\lambda t\right\} .
$$

In the first case, since $Q_{t, j}$ s are pairwise disjoint,

$$
\sum_{\left\{j: Q_{t, j} \in Q_{0} \subset\left\{f^{\# 1}>\lambda t\right\}\right\}} v\left(Q_{t, j}\right) \leq v\left(\left\{f^{\#^{1}}>\lambda t\right\}\right),
$$


and also,

$$
\sum_{\substack{Q_{0} \\ Q_{0} \subset\left\{f^{1}>\lambda t\right\}}} \sum_{\left\{j: Q_{t, j} \subset Q_{0}\right\}} v\left(Q_{t, j}\right) \leq v\left(\left\{f^{\# 1}>\lambda t\right\}\right) .
$$

In the second case,

$$
\frac{1}{\sigma\left(Q_{0}\right)} \int_{Q_{0}}\left|f-f_{Q_{0}}\right| d \sigma \leq \lambda t
$$

Since $2^{-1} t>\|f\|_{L^{1}(S)}$, by (4.5), taking $f_{\mathrm{Q}_{0}} \leq R_{2} k t=2^{-1} t$ into account, we have

$$
\begin{aligned}
\sum_{\left\{j: Q_{t, j} \in Q_{0} \not \subset\left\{f^{\sharp 1}>\lambda t\right\}\right\}}(t-t / 2) \sigma\left(Q_{t, j}\right) & \leq \sum_{\left\{j: Q_{t, j} \in Q_{0} \not \subset\left\{f^{f 1}>\lambda t\right\}\right\}} \int_{Q_{t, j}} f-f_{Q_{0}} d \sigma \\
& \leq \sum_{\left\{j: Q_{t, j} \subset Q_{0} \not \subset\left\{f^{f 1}>\lambda t\right\}\right\}} \int_{Q_{t, j}}\left|f-f_{Q_{0}}\right| d \sigma \\
& \leq \int_{Q_{0}}\left|f-f_{Q_{0}}\right| d \sigma \\
& \leq \lambda t \sigma\left(Q_{0}\right) \quad(\text { by (4.8)). }
\end{aligned}
$$

Thus,

$$
\sum_{\left.\left\{j: Q_{t, j} \subset Q_{0} \not \subset f^{\sharp 1}>\lambda t\right\}\right\}} \sigma\left(Q_{t, j}\right) \leq 2 \lambda \sigma\left(Q_{0}\right) .
$$

In (4.9), take a small $\lambda>0$ such that

$$
2 \lambda<1 .
$$

(Note that the condition (4.10) enables us to use (1.3).) Thus, (4.9) can be written as

$$
\sum_{\left\{j: Q_{t, j} \subset Q_{0} \not \subset\left\{f^{\# 1}>\lambda t\right\}\right\}} v\left(Q_{t, j}\right) \leq\left(1-(1-2 \lambda)^{p}\right) v\left(2 Q_{\kappa t, j_{0}}\right) .
$$

Adding up all possible $Q_{0}$ 's in the second case of (4.6), we get

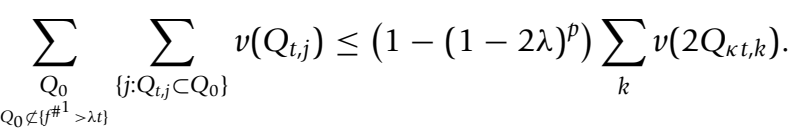

Since $\{M f>t\} \subset\left\{M f>R_{2}^{-1} t\right\}$ and $\sigma\left(2 Q_{t, j}\right) \leq R_{2} t^{-1} \int_{2 Q_{t, j}} f d \sigma$ holds (4.5), we can construct the collection of balls $\left\{Q_{R_{2}^{-1} t, j}\right\}$ which covers $\left\{M f>R_{2}^{-1} t\right\}$ with maximal radius just the same way as Lemma 4.1 , so that $2 Q_{t, j}$ is contained in $\left\{Q_{R_{2}^{-1} t, i}\right\}$ for some $i$. Recall that $R_{2}^{-1} k t=2^{-1} R_{2}^{-2} t>\|f\|_{L^{1}(S)}$, hence, (4.11) turns into

$$
\sum_{\substack{Q_{0} \\ Q_{0}\left\langle\left\langle u^{+1}>\lambda t\right\}\right.}} \sum_{\left\{j: Q_{t, j} \subset Q_{0}\right\}} v\left(Q_{t, j}\right) \leq\left(1-(1-2 \lambda)^{p}\right) \sum_{k} v\left(Q_{R_{2}^{-1} \kappa t, k}\right) .
$$


Combining (4.7) and (4.11), we summarize

$$
\sum_{j} v\left(Q_{t, j}\right) \leq v\left(\left\{f^{\#^{1}}>\lambda t\right\}\right)+\sum_{k}\left(1-(1-2 \lambda)^{p}\right) v\left(Q_{R_{2}^{-1} \kappa t, k}\right) .
$$

Now, put

$$
\begin{aligned}
& \alpha_{v}(t)=\sum_{j} v\left(Q_{t, j}\right), \\
& \beta_{u}(t)=u\left(E_{t}\right) .
\end{aligned}
$$

Then,

$$
\begin{aligned}
\beta_{u}(t) & \leq \int_{\cup_{j} 3 Q_{t, j}} u d \sigma \leq \sum_{j} \int_{3 Q_{t, j}} u d \sigma \\
& \left.\leq A_{p} \sum_{j} \int_{3 Q_{t, j}} v d \sigma \quad \text { (by Corollary 2.2 with } E=Q=3 Q_{t, j}\right) \\
& \leq A_{p} \sum_{j} \int_{\left.Q_{R_{3}^{-1}}\right)} v d \sigma \\
& =A_{p} \alpha_{v}\left(R_{3}^{-1} t\right),
\end{aligned}
$$

where the fourth inequality follows from the fact that $3 Q_{t, j} \subset Q_{R_{3}^{-1} t, i}$ for some $i$. Indeed, we can construct $Q_{R_{3}^{-1} t, i}$ as before, since $R_{3}^{-1} t>\|f\|_{L^{1}(S)}$.

Eventually, putting the constant $e_{p}=\int_{0}^{\infty} t^{p} e^{-t} d t$, and $N=\max \left(2,2 R_{2}^{2}, R_{3}\right)$ (which depends only on $n$ ), we have

$$
\begin{aligned}
\int_{S}|M f|^{p} u d \sigma & \leq \int_{0}^{\infty} p t^{p-1} \beta_{u}(t) d t+e_{p} \varepsilon \\
& \leq \int_{0}^{N\|f\|_{L^{1}(S)}} p t^{p-1} \beta_{u}(t) d t+A_{p} \int_{N\|f\|_{L^{1}(S)}}^{\infty} p t^{p-1} \alpha_{\nu}\left(R_{3}^{-1} t\right) d t+e_{p} \varepsilon \quad(\text { by (4.15)) } \\
& :=I+I I+e_{p} \varepsilon
\end{aligned}
$$

The first term $I$ is dominated by

$$
\begin{aligned}
N^{p}\|u\|_{L^{1}(S)}\|f\|_{L^{1}(S)}^{p} & \leq N^{p}\|f\|_{L^{p(v)}}^{p}\|u\|_{L^{1}(S)}\left(\int_{S} v^{-1 /(p-1)} d \sigma\right)^{p-1} \\
& \leq N^{p} A_{p}\|f\|_{L^{p(v)}}^{p} \quad\left(\text { since }(u, v) \in A_{p}(S)\right),
\end{aligned}
$$

where the first inequality follows from Hölder's inequality for $\|f\|_{L^{1}(S)}^{o}$.

On the other hand,

$$
\begin{aligned}
I I & \leq A_{p} C_{p} \int_{0}^{\infty} p t^{p-1} v\left(\left\{f^{\#^{1}}>R_{3}^{-1} t\right\}\right) d t \quad \text { (by Lemma 4.3) } \\
& =A_{p} C_{p} R_{3}^{p} \int_{0}^{\infty} p t^{p-1} v\left(\left\{f^{\#^{1}}>t\right\}\right) d t \quad \text { (by the change of variable) } \\
& =A_{p} C_{p} R_{3}^{p} \int_{S}\left|f^{\# 1}\right|^{p} v d \sigma .
\end{aligned}
$$


Hence,

$$
\int_{S}|M f|^{p} u d \sigma \leq N^{p} A_{p}\|f\|_{L^{p}(v)}^{p}+A_{p} C_{p} R_{3}^{p} \int_{S}\left|f^{\#^{1}}\right|^{p} v d \sigma+e_{p} \varepsilon .
$$

The first and the last integrals are independent of $\varepsilon$. Letting $\varepsilon \searrow 0$, therefore, the proof is complete after accepting Lemma 4.3.

Lemma 4.2. Let $\alpha_{v}$ be defined in (4.14). Then, for every $q \geq p$ and every $r>0$,

$$
\int_{0}^{r} t^{q-1} \alpha_{v}(t) d t<\infty
$$

Proof. For a positive real number $r$, we set

$$
I_{r}=\int_{0}^{r} q t^{q-1} \alpha_{v}(t) d t
$$

Since $\sum_{j} v\left(Q_{t, j}\right) \leq \int_{\{M f>t\}} v d \sigma$, we have

$$
I_{r} \leq \int_{0}^{r} q t^{q-1} \int_{\{M f>t\}} v d \sigma d t
$$

We note that $I_{r}$ is finite, since $p \geq p_{0}$ and it is bounded by

$$
\frac{q r^{q-p}}{p} \int_{0}^{r} p t^{p-1} \int_{\{M f>t\}} v d \sigma d t \leq \frac{q r^{q-p}}{p}\|M f\|_{L^{p}(v)}^{p}<\infty,
$$

since $M f \in L^{p}(v)$. Therefore, the proof is complete.

Now, filling up next lemma, we finish the proof of Theorem 1.2.

Lemma 4.3. Under the same assumption as Theorem 1.2, if $\alpha_{v}$ is defined in (4.14), then there is a constant $C_{p}$ such that

$$
\int_{0}^{\infty} t^{p-1} \alpha_{v}(t) d t \leq C_{p} \int_{0}^{\infty} t^{p-1} v\left(\left\{f^{\#^{1}}>t\right\}\right) d t .
$$

Proof. Recall (4.13), i.e.,

$$
\alpha_{v}(t) \leq v\left(\left\{f^{\#^{1}}>\lambda t\right\}\right)+\left(1-(1-2 \lambda)^{p}\right) \alpha_{v}\left(R_{2}^{-1} \kappa t\right) .
$$

By integration, it follows that

$$
\begin{aligned}
& \int_{0}^{r} t^{p-1} \alpha_{v}(t) d t \\
& \leq \int_{0}^{r} t^{p-1} v\left(\left\{f^{\# 1}>\lambda t\right\}\right) d t \\
& \quad+\left(1-(1-2 \lambda)^{p}\right) \int_{0}^{r} t^{p-1} \alpha_{v}\left(R_{2}^{-1} k t\right) d t \\
& =\int_{0}^{r} t^{p-1} v\left(\left\{f^{\# 1}>\lambda t\right\}\right) d t \\
& \quad+\left(1-(1-2 \lambda)^{p}\right) R_{2}^{p} k^{-p} \int_{0}^{R_{2}^{-1} k r} t^{p-1} \alpha_{v}(t) d t \\
& \leq \int_{0}^{r} t^{p-1} v\left(\left\{f^{\# 1}>\lambda t\right\}\right) d t \\
& \quad+2^{p} R_{2}^{2 p}\left(1-(1-2 \lambda)^{p}\right) \int_{0}^{r} t^{p-1} \alpha_{v}(t) d t \quad\left(\text { since } k=2^{-1} R_{2}^{-1}, R_{2}^{-1} k<1\right),
\end{aligned}
$$


where the equality is due to the change of variable.

Take a small $\lambda$ so that

$$
\begin{aligned}
2^{p} R_{2}^{2 p}\left(1-(1-2 \lambda)^{p}\right) & <1 / 2 \\
2 \lambda & <1
\end{aligned}
$$

where the second inequality comes from (4.10). Then, by Lemma 4.2, (4.17) can be written as

$$
\begin{aligned}
\frac{1}{2} \int_{0}^{r} t^{p-1} \alpha_{v}(t) d t & \leq \int_{0}^{r} t^{p-1} v\left(\left\{f^{\# 1}>\lambda t\right\}\right) d t \\
& =\lambda^{-p} \int_{0}^{\lambda r} t^{p-1} v\left(\left\{f^{\# 1}>t\right\}\right) d t,
\end{aligned}
$$

where the equality is caused by the change of variable.

Finally, letting $r \rtimes \infty$, we obtain

$$
\int_{0}^{\infty} t^{p-1} \alpha_{v}(t) d t \leq 2 \lambda^{-p} \int_{0}^{\infty} t^{p-1} v\left(\left\{f^{\#^{1}}>t\right\}\right) d t
$$

Therefore, the proof is complete.

\section{Acknowledgements}

We would like to express our deep gratitude to the referee for careful suggestions. His suitable and valuable comments were able to make a great improvement of the original manuscript.

\section{Authors' contributions}

KSR drove a sufficient condition for two-weighted norm inequalities for $K$. In proving cross-weighted norm inequalities between the Hardy-Littlewood maximal function and the sharp maximal function on the unit sphere, and $J$ carried out the study about the covering lemma on the sphere. All authors read and approved the final manuscript.

\section{Competing interests}

The author Kyung Soo Rim was supported in part by a National Research Foundation of Korea Grant, NRF 20110027339 .

Received: 25 March 2011 Accepted: 22 November 2011 Published: 22 November 2011

\section{References}

1. Lee, J, Rim, KS: Properties of M-harmonic conjugate operator. Canad Math Bull. 46, 113-121 (2003). doi:10.4153/CMB2003-011-x

2. Rudin, W: Function Theory in the Unit Ball of $C^{n}$. Springer, New York (1980)

3. Garnett, JB: Bounded Analytic Functions. Academic Press, New York (1981)

4. Hunt, R, Muckenhoupt, B, Wheeden, R: Weighted norm inequalities for the conjugate function and Hilbert transform. Trans Amer Math Soc. 176, 227-251 (1973)

5. Lee, J, Rim, KS: Estimate of M-harmonic conjugate operator. J Inequal Appl 13 (2010). Art. ID 435450

6. Muckenhoupt, B: Two weight function norm inequalities for the Poisson integral. Trans Amer Math Soc. 210, 225-231 (1975)

7. Sawyer, ET: Two weight norm inequalities for certain maximal and integral operators. In Harmonic Analysis (Minneapolis, MN, 1981), Lecture Notes in Mathematics, vol. 908,Springer, Berlin (1982)

8. Garcia-Cuerva, J, Rubio, JL: Weighted norm inequalities and related topics. In North-Holland Mathematics Studies, vol. 116, North-Holland, New York (1985)

9. Stein, EM: Harmonic Analysis, Real Variable Methods, Orthogonality, and Oscillatory Integrals. Princeton University Press, Princeton (1993)

10. Grafakos, L: Classical and Modern Fourier Analysis. Pearson Education, Inc., Upper Saddle River (2004)

11. Muckenhoupt, B, Webb, DW: Two-weight norm inequalities for Cesàro means of Laguerre expansions. Trans Amer Math Soc. 353, 1119-1149 (2001). doi:10.1090/50002-9947-00-02729-X

12. Muckenhoupt, B, Webb, DW: Two-weight norm inequalities for the Cesàro means of Hermite expansions. Trans Amer Math Soc. 354, 4525-4537 (2002). doi:10.1090/50002-9947-02-03093-3

13. Garcia-Cuerva, J, Martell, JM: Two-weight norm inequalities for maximal operators and fractional integrals on nonhomogeneous spaces. Indiana Univ Math. J. 50, 1241-1280 (2001)

14. Pérez, C: Two weighted norm inequalities for Riesz potentials and uniform $L^{p}$-weighted Sobolev inequalities. Indiana Univ Math J. 39, 31-44 (1990). doi:10.1512/iumj.1990.39.39004

15. Pérez, C: Two weighted inequalities for potential and fractional type maximal operators. Indiana Univ Math J. 43, 663-683 (1994). doi:10.1512/iumj.1994.43.43028 
16. Li, W: Two-weight norm inequalities for commutators of potential type integral operators. J Math Anal Appl. 322, 1215-1223 (2006). doi:10.1016/j.jmaa.2005.08.051

17. Zani, SL: Two-weight norm inequalities for maximal functions on homogeneous spaces and boundary estimates. Studia Math. 126, 67-94 (2007)

18. Sawyer, ET: A characterization of a two-weight norm inequality for maximal operators. Studia Math. 75, 1-11 (1982)

19. Sawyer, ET: A characterization of two weight norm inequalities for fractional and Poisson integrals. Trans Amer Math Soc. 308, 533-545 (1988). doi:10.1090/S0002-9947-1988-0930072-6

20. Neugebauer, CJ: Inserting $A_{p}$-weights. Proc Amer Math Soc. 87, 644-648 (1983)

21. Cruz-Uribe, D, Pérez, C: Sharp two-weight, weak-type norm inequalities for singular integral operators. Math Res Lett. 6 , 417-427 (1999)

22. Martell, J, Cruz-Uribe, D, Pérez, C: Sharp two-weight inequalities for singular integrals, with applications to the Hilbert transform and the Sarason conjecture. Adv Math. 216, 647-676 (2007). doi:10.1016/j.aim.2007.05.022

23. Ding, Y, Lin, CC: Two-weight norm inequalities for the rough fractional integrals. Int J Math Math Sci. 25, 517-524 (2001). doi:10.1155/S0161171201003982

24. Muckenhoupt, B, Wheeden, RL: Two weight function norm inequalities for the Hardy-Littlewood maximal function and the Hillbert transform. Studia Math. 55, 279-294 (1976)

doi:10.1186/1029-242X-2011-117

Cite this article as: Rim and Lee: Norm inequalities for the conjugate operator in two-weighted Lebesgue spaces. Journal of Inequalities and Applications 2011 2011:117.

\section{Submit your manuscript to a SpringerOpen ${ }^{\odot}$} journal and benefit from:

Convenient online submission

- Rigorous peer review

- Immediate publication on acceptance

- Open access: articles freely available online

- High visibility within the field

- Retaining the copyright to your article

Submit your next manuscript at $\boldsymbol{s p r i n g e r o p e n . c o m ~}$ 